University of Nebraska - Lincoln

DigitalCommons@University of Nebraska - Lincoln

\title{
Least-cost Disjoint Paths with Dependent Cost Structure in Wavelength Continuous Optical WDM Networks
}

\author{
Ajay Todimala \\ University of Nebraska-Lincoln, ajayt@cse.unl.edu \\ Byrav Ramamurthy \\ University of Nebraska-Lincoln, bramamurthy2@unl.edu
}

Follow this and additional works at: https://digitalcommons.unl.edu/cseconfwork

Part of the Computer Sciences Commons

Todimala, Ajay and Ramamurthy, Byrav, "Least-cost Disjoint Paths with Dependent Cost Structure in Wavelength Continuous Optical WDM Networks" (2005). CSE Conference and Workshop Papers. 98. https://digitalcommons.unl.edu/cseconfwork/98 


\title{
Least-cost Disjoint Paths with Dependent Cost Structure in Wavelength Continuous Optical WDM Networks
}

\author{
Ajay Todimala and Byrav Ramamurthy \\ Department of Computer Science and Engineering \\ University of Nebraska-Lincoln \\ Lincoln NE 68588-0115 U.S.A. \\ Email: $\{$ ajayt, byrav $\} @$ cse.unl.edu
}

\begin{abstract}
One of the important issues in establishing a fault tolerant connection in a wavelength division multiplexing optical network is computing a pair of disjoint working and protection paths and a free wavelength along the paths. While most of the earlier research focused only on computing disjoint paths, in this work we consider computing both disjoint paths and a free wavelength along the paths.

The concept of dependent cost structure (DCS) of protection paths to enhance their resource sharing ability was proposed in our earlier work. In this work we extend the concept of DCS of protection paths to wavelength continuous networks. We formalize the problem of computing disjoint paths with DCS in wavelength continuous networks and prove that it is NP-complete. We present an iterative heuristic that uses a layered graph model to compute disjoint paths with DCS and identify a free wavelength.
\end{abstract}

\section{INTRODUCTION}

The problem of providing fault-tolerance to wavelength division multiplexing (WDM) optical networks has enjoyed a lot of attention in recent times. Shared protection is a popularly known technique to provide fault-tolerance to optical WDM networks. It allows protection paths of more than one connection to share resources when their corresponding working paths do not fail at the same time. If the working path of a newly arrived connection is disjoint with the working path of an active connection in the network, then a wavelength-link that is reserved for protecting the active connection can be used by the protection path of the newly arrived connection request at no extra cost. This technique of providing shared protection is popularly known as backup multiplexing.

An optimal protection path under shared protection is a path that utilizes as much resources as possible from the pool of resources that are reserved for protecting currently active connections in the network. To compute such an optimal path one needs to consider the following two criteria. First, the set of currently active connections in the network whose working paths are edge-disjoint with the working path of the newly arrived connection. Second, the wavelength-links (network resources) that are reserved for protecting the active connections that satisfy the first criteria. We term this dependency of the protection path as dependent cost structure of protection paths under shared protection in wavelength continuous networks.

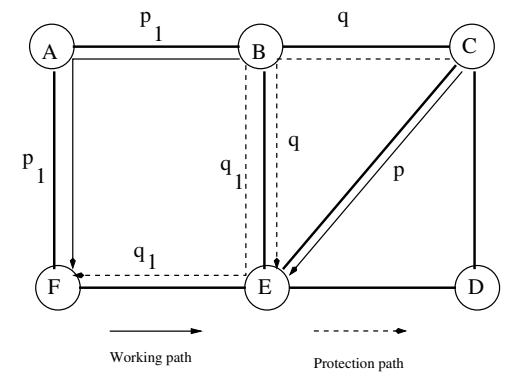

Fig. 1. Illustration of dependent cost structure of protection paths in wavelength continuous networks.

Figure 1 shows a simple 6 -node wavelength continuous optical network. Connection $c_{1}$ between node pair $(B, F)$ is established on working and protection path pair $\left(p_{1}, q_{1}\right)$ on wavelength $\lambda_{1}$. A new connection request $c$ between node pair $(C, E)$ arrives. The working path $p$ for the new connection request is established on the link $\overrightarrow{C E}$ and on wavelength $\lambda_{1}$. Without considering the dependent cost structure, the costs of the paths $\overrightarrow{C B E}$ and $\overrightarrow{C D E}$ are the same, assuming all the links have equal cost. Now considering the dependent cost structure, since the paths $p_{1}$ and $p$ are disjoint, the link $\overrightarrow{B E}$ can be used by the protection path of the connection $c$ at no extra cost i.e., dependent cost of link $\overrightarrow{B E}$ is zero. Therefore path $\overrightarrow{C B E}$ is chosen as the protection path of connection $c$. In this way dependent cost structure allows the protection path of the newly arrived connection to share as much resources as possible from the pool of resources that are reserved for protecting currently active connections in the network and thus enhances the resource sharing ability of the protection paths.

The dependent cost structure optimizes the reserved capacity both in static and dynamic network environments. 
In a dynamic environment, where connection requests are not known, dependent cost structure provides improved blocking performance and resource utilization. In a static environment, where connections requests are known ahead of time, it minimizes the amount of resources required to establish the given set of connections or maximize the number of connections that can be established under resource constraints. In both cases it provides increased revenue for service providers. Dependent cost structure of protection paths without considering wavelength availability was studied in our earlier work [1]. The problem was proved to be NP-complete and hard to approximate. However our earlier work did not consider wavelength availability along the computed disjoint routes. In this work we consider both wavelength availability along the disjoint working and protection paths and dependent cost structure of the protection paths.

The rest of the paper is organized as follows. In Section II we formalize the least-cost disjoint paths problem with wavelength continuity constraint and dependent cost structure (LDP-WCC-DCS). In Section III we present our iterative heuristic for solving the LDP-WCC-DCS problem. In Section IV we discuss the experimental results and in Section V we present our concluding remarks.

\section{Problem Formulation}

In this section we define the dependent cost of a path $q$ (protection path) w.r.t. the path $p$ (working path) and the current status of the graph $G$ with $\kappa$ active connections. We provide a formal definition of LDP-WCC-DCS problem and prove that it is NP-complete.

\section{A. Preliminaries}

In this section we introduce few notations that are later used in the formulation of the problem. We follow the convention that the path $p_{i}$ in the ordered pair $\left(p_{i}, q_{i}\right)$ is the working path and the path $q_{i}$ is the protection path and $\left(\lambda_{p_{i}}, \lambda_{q_{i}}\right)$ are the wavelengths assigned to paths $p_{i}$ and $q_{i}$ respectively.

A path $p$ is said to be link disjoint with path $q$ if there exists no common link between them, and is denoted as $p \cap q=\phi$. A wavelength-link in a optical fiber graph $G=(V, E)$ with $W$ wavelengths, i.e., link $(i, j)$ on wavelength $\lambda$, is denoted as $((i, j), \lambda)$. A path $p$ along with wavelength assignment $\lambda_{p}$ is said to be wavelengthlink disjoint with a set of paths $P$ and their corresponding wavelength assignments $\Lambda_{P}$ if path $p$ does not use any wavelength-link that is assigned to any of the paths in the set $P$, and is denoted as $\left[\left(p, \lambda_{p}\right) \cap\left(P, \Lambda_{P}\right)\right]=\phi$. The wavelength-link disjointedness can be mathematically formulated as,

$$
\begin{array}{r}
{\left[\left(p, \lambda_{p}\right) \cap\left(P, \Lambda_{P}\right)\right]=\phi \Longleftrightarrow} \\
\forall p_{i} \in P, \text { if }((i, j) \in p) \wedge\left((i, j) \in p_{i}\right) \text { then } \lambda_{p} \neq \lambda_{p_{i}} .
\end{array}
$$

\section{B. Dependent Cost Structure}

In this section we formalize the dependent cost structure of a protection path on its working path and the current status of the network with $\kappa$ active connections. The dependent cost of a wavelength-link $((i, j), \lambda)$, $C_{\left(P, Q, \Lambda_{Q}, p\right)}(i, j, \lambda)$, in a graph $G$ with $\kappa$ disjoint path pairs $(P, Q)$ already routed on wavelengths $\left(\Lambda_{P}, \Lambda_{Q}\right)$ and with a working path $p$ between the node pair $(s, t)$ is defined as follows: $C_{\left(P, Q, \Lambda_{Q}, p\right)}((i, j), \lambda)$

$$
=\begin{array}{ll}
O, & \text { if } \exists q_{i} \in Q, \text { s. t. }\left((i, j) \in q_{i}\right) \\
& \wedge\left(\lambda_{q_{i}}=\lambda\right) \wedge\left(\left(p_{i} \cap p\right)=\phi\right) \\
C((i, j), \lambda), & \text { otherwise }
\end{array}
$$

where $C((i, j), \lambda)$ is the cost of link $(i, j)$ on wavelength $\lambda$. The dependent cost of path $q$ w.r.t. path $p$ in the graph with $\kappa$ pairs of disjoint working and protection paths already routed is sum of dependent costs of all the links along the path $q$ and is given by the following equation.

$$
C_{\left(P, Q, \Lambda_{Q}, p\right)}(q, \lambda)=\sum_{(i, j) \in q} C_{\left(P, Q, \Lambda_{Q}, p\right)}((i, j), \lambda)
$$

\section{Formulation}

Let us formalize the least-cost disjoint paths problem with wavelength continuity constraint and dependent cost structure (LDP-WCC-DCS).

\section{Least-cost Disjoint Paths problem with Wavelength Continuity Constraint and Dependent Cost Structure (LDP-WCC-DCS)}

Instance: Given a network topology graph $G=(V, E)$, an integer cost function $C, \kappa$ pairs of edge disjoint paths $\left(p_{i}, q_{i}\right)$ routed on wavelengths $\left(\lambda_{p_{i}}, \lambda_{q_{i}}\right)$ corresponding to $\kappa$ node pairs $\left(s_{i}, t_{i}\right)$ where $1 \leq \lambda_{p_{i}}, \lambda_{q_{i}} \leq W$, a sourcedestination node pair $(s, t)$, and an integer $M$.

Question: Does there exist an edge-disjoint path pair $(p, q)$ and wavelength assignment $\left(\lambda_{p}, \lambda_{q}\right)$ between node pair $(s, t)$ such that $\left[\left(p, \lambda_{p}\right) \cap\left(P, \Lambda_{P}\right)\right]=\phi,\left[\left(p, \lambda_{p}\right) \cap\right.$ $\left.\left(Q, \Lambda_{Q}\right)\right]=\phi,\left[\left(q, \lambda_{q}\right) \cap\left(P, \Lambda_{P}\right)\right]=\phi$ and its cost $C(p, q)=C(p)+C_{\left(P, Q, \Lambda_{Q}, p\right)}(q)$ is at most $M$ where $C(p)$ is the cost of path $p$ and $C_{\left(P, Q, \Lambda_{Q}, p\right)}(q)$ is the dependent cost of $q$ w.r.t. to $\left(P, Q, \Lambda_{Q}, p\right)$ ?

The least-cost disjoint paths problem (LDP-DCS) without considering wavelength continuity constraint but with dependent cost structure was proved NP-complete and hard to approximate in [1]. LDP-WCC-DCS easily reduces to LDP-DCS problem and is therefore NP-complete. In the next section we present our heuristic that uses the layered graph model to compute the least-cost disjoint path pair in wavelength continuous networks considering dependent cost structure. 


\section{OUR HEURISTICS}

In our earlier work in [2] we presented an iterative heuristic IMNH to compute asymmetrically-weighted leastcost disjoint paths. In this section we extend the IMNH heuristic to IMNH-LWD heuristic for solving the LDPWCC-DCS problem. IMNH-LWD computes routes as well as free wavelength along the routes by computing the routes in a layered graph [3] instead of regular fiber topology graph. Though the LDP-WCC-DCS problem formulation and the NP-completeness results presented in Section II are only applicable for networks without wavelength conversion, the IMNH-LWD heuristic presented in this section can be used for networks without wavelength conversion as well as with limited wavelength conversion.

\section{A. Our Iterative Heuristic (IMNH-LWD) for LDP-WCC- DCS Problem}

In this section we present our iterative heuristic for computing least-cost disjoint path pair with dependent cost structure in layered graph (IMNH-LWD). The IMNH-LWD heuristic is described in the flow-chart in Figure 2. The input to the heuristic is a fiber topology represented by graph $G$, its edge cost function $C$, the layered graph $G_{L}$ corresponding to graph $G, \kappa$ pairs of working and protection path pairs $(P, Q)$ corresponding to $\kappa$ active connections, their wavelength assignments given by $\Lambda_{P}, \Lambda_{Q}$, the edge cost function $C_{G_{L}}$ of layered graph $G_{L}$, and the source destination node pair $(s, t)$. The transformation of given graph $G=(V, E)$ to layered graph $G_{L}=\left(V_{L}, E_{L}\right)$, $\Gamma: G \longrightarrow G_{L}$ is presented in Appendix A. A shortest path in this layered graph computes both a path and a wavelength assignment along the path. The edge cost function $C_{G_{L}}$ reflects the state of the network with $\kappa$ active connections.

The iterative heuristic IMNH-LWD, similar to IMNH$\mathrm{WD}$, iteratively tries to compute the disjoint paths and minimize its cost. During its computation, IMNH-LWD maintains the current best disjoint path pair $P P_{\text {cur-opt }}$ found which is initialized to NULL. The number of iterations of the IMNH-LWD heuristic is limited by a predetermined integer $M$. Starting with the shortest path as the seed path in its first iteration, it uses next shortest path with non-decreasing cost as seed path in each successive iteration. IMNH-LWD using the modified network-flow algorithm MNA and the $i^{t h}$ shortest path $s_{i}$ as seed path computes a pair of disjoint paths $(p, q)$ in $G$. The MNA algorithm is described in Section III-B. The IMNH-LWD heuristic now invokes TSH-DL assuming the seed path $p$ as working path to compute the disjoint protection path $p^{\prime}$ in $G_{L}$. The TSH-DL heuristic is described in Section III-C. If the path pair computed in the $i^{\text {th }}$ iteration $\left(p, p^{\prime}\right)$ is better than the current best optimal path pair $P P_{c u r-o p t}$, it updates the path pair $P P_{\text {cur-opt }}$ as $\left(p, p^{\prime}\right)$. Now, the heuristics checks to see if the current best optimal $P P_{\text {cur-opt }}$ is the

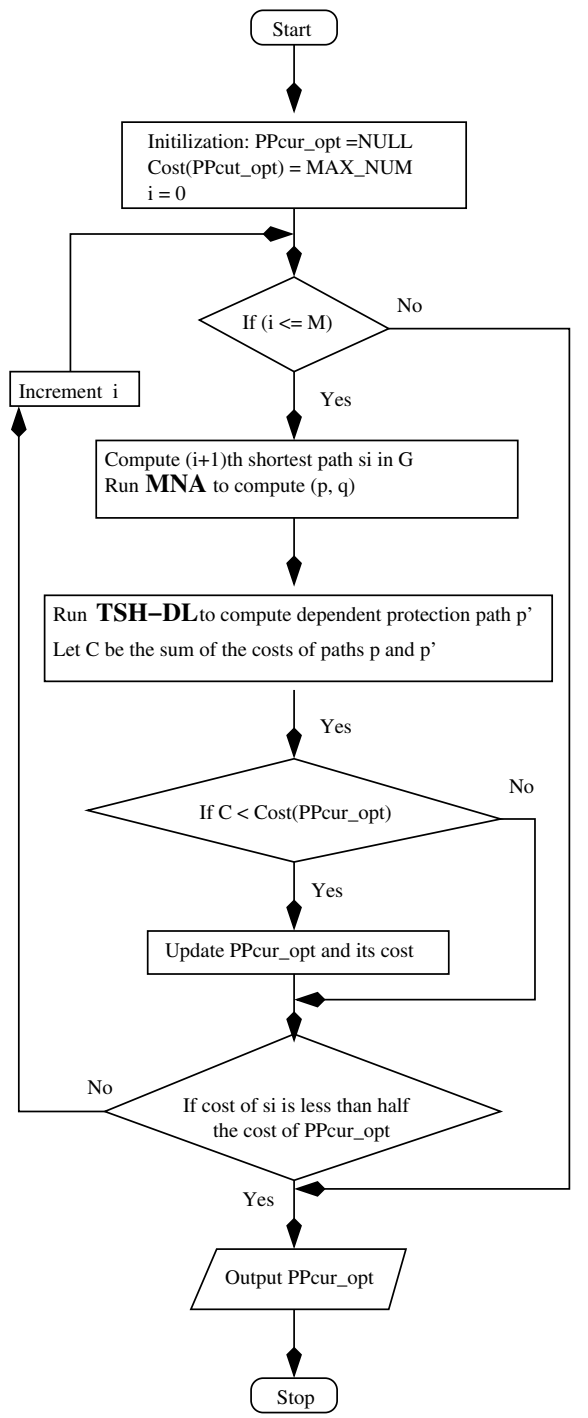

Fig. 2. The flow-chart of the Iterative Modified Network-flow Heuristic for solving LDP-WCC-DCS problem (IMNH-LWD).

optimal solution. If IMNH-LWD heuristic successfully verifies the optimality of the path pair, it stops and outputs the optimal disjoint path pair, otherwise it continues with the next iteration.

If the optimality of the path pair $P P_{c u r-o p t}$ could not be established even after completing $M$ iterations, IMNHLWD returns the current best solution as a sub-optimal solution. A measure of closeness of the optimality of the sub-optimal solution to the optimal solution is given by Theorem 1.

Theorem 1: Let $P P_{\text {cur-opt }}$ be the current optimal disjoint path pair computed by IMNH-AWD algorithm at the end of $i^{t h}$ iteration, $C_{\text {cur-opt }}$ be its weighted cost, let $C\left(s_{i}\right)$ be the cost of the $i^{t h}$ shortest path in graph $G$ and optimal not found then $C_{\text {cur-opt }}$ is at most $\frac{C_{c u r-o p t}}{(\alpha+1) C\left(s_{i}\right)}$ times 
optimal solution.

Proof: Let opt be cost of the optimal disjoint path pair $(p, q)$ i.e., opt $=\alpha p+q$. Now, opt $\geq \alpha C\left(s_{i}\right)+C\left(s_{i}\right)$, since if opt $<\alpha C\left(s_{i}\right)+C\left(s_{i}\right)$ the optimal path pair $(p, q)$ must have already been computed in some iteration $j, j<i$ where $C\left(s_{j}\right)<C\left(s_{i}\right)$, a contradiction.

Now consider the ratio,

$$
\begin{aligned}
\frac{C_{\text {cur-opt }}}{o p t} & \leq \frac{C_{\text {cur-opt }}}{(\alpha+1) C\left(s_{i}\right)} \\
C_{\text {cur-opt }} & \leq \frac{C_{\text {cur-opt }}}{(\alpha+1) C\left(s_{i}\right)} \times o p t .
\end{aligned}
$$

\section{B. Modified Network-flow Algorithm (MNA) for Layered-} Graph

In this section we present modified network-flow algorithm (MNA), outlined in Algorithm 1. The input to the algorithm is a directed graph $G$, an edge-cost function $C$, and the seed path $p$ between the source-destination pair $(s, t)$. The algorithm computes a pair of edge disjoint paths, if such a disjoint path pair exists in the graph, otherwise it returns NULL.

The trap links along a path $s_{k}$ are defined as the links along path $s_{k}$ that are responsible for the failure of the TSH heuristic in computing disjoint path pair. The twostep heuristic (TSH) is a simple heuristic to compute a pair of edge disjoint paths between a pair of nodes in a graph $G$. The MNA algorithm computes a pair of disjoint paths, if a diverse path pair can be computed by avoiding trap links along the seed path $s_{k}$, otherwise it returns NULL. To identify trap links along the given seed path $s_{k}$, MNA, in steps $2-3$, constructs a modified graph $G^{\prime}$ and its cost function $C^{\prime}$ as follows. It removes the directed edges $(i, j)$ along the seed path $s_{k}$ and assigns the cost of link $(j, i)$ to 0 . Now, MNA checks to see if a shortest path $s_{k}^{\prime}$ between node pair $(s, t)$ exists in the modified graph $G^{\prime}$. If $s_{k}^{\prime}$ exists then, MNA successfully computes the disjoint paths in steps $5-9$ and outputs sum of their costs, otherwise MNA fails and returns $\infty$. The trap links along the seed path $s_{k}$ are the links that are belong to both the path $s_{k}$ and $s_{k}^{\prime}$ (ignoring the link directions). In Steps $5-6$, MNA adds all the links along the paths $s_{k}$ and $s_{k}^{\prime}$ except the trap links to another temporary graph $G^{\prime \prime}$. In step 7, MNA computes link disjoint paths $(p, q)$ and in step 8 it returns the cost of the pair $(p, q)$.

1) Complexity: Let us analyze the complexity of the MNA algorithm. Steps $2-3$ take $O(n)$ time, assuming that the seed path has no cycles. Dijkstra's algorithm can be used to compute the shortest path $s_{k}^{\prime}$ in Step 4 . We know that the time complexity of Dijkstra's algorithm is $O(m+n \log n)$ where $n$ is the number of nodes and $m$ is the number of links in the network. Steps $5-6$ can be computed in $O\left(n^{2}\right)$. In Step 7 the MNA can use TSH approach to compute the

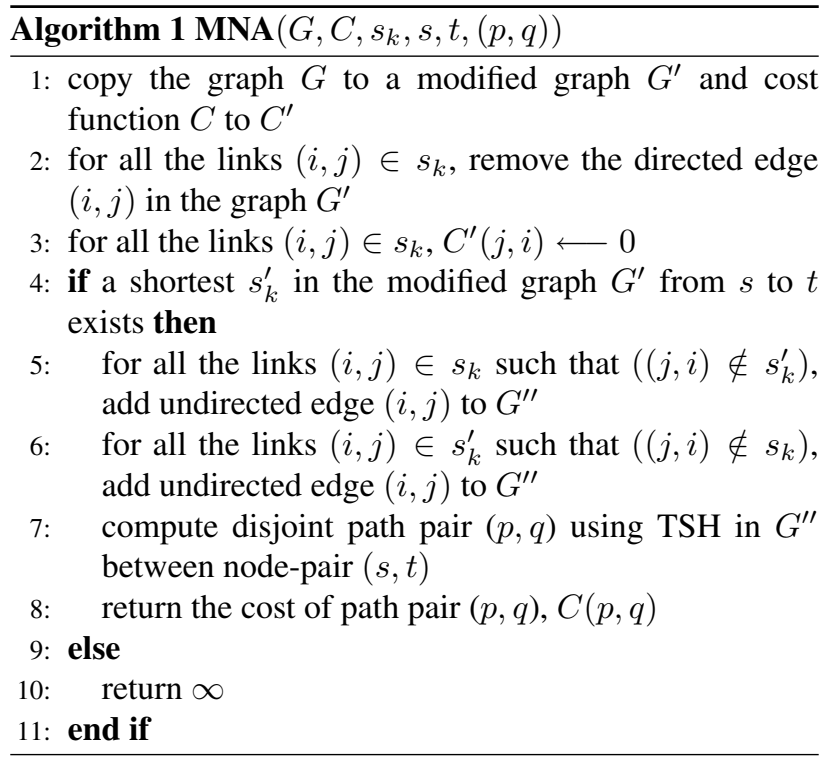

diverse paths in $G^{\prime \prime}$. The complexity of Step 7 is again $O(m+n \log n)$. Therefore the time complexity of the MNA algorithm is $O\left(m+n \log n+n^{2}\right)$.

Note that the paths computed in Step 7 use only the links along the seed path $s_{k}$ and path $s_{k}^{\prime}$ but not the links belong to both the paths. From this observation Step 7 can be enhanced to compute the diverse paths by a simple rearrangement in $O(n)$ time. With this enhancement, like TSH, MNA invokes Dijkstra's algorithm only once. Hence the running times of TSH and MNA can be considered equivalent.

\section{Two-Step Heuristic with Dependent cost structure using the Layered graph model (TSH-DL)}

In this section we present the two-step heuristic for computing the edge disjoint protection path with dependent cost structure assuming the given seed path as the working path where the input graph is a layered graph. The two-step heuristic considering dependent cost structure for layered graph (TSH-DL) is outlined in Algorithm 2. The input to the TSH-DL heuristic is same as IMNH-LWD including a seed path $p$. The heuristic computes the protection path $q$ in the layered graph and returns the weighted sum of the costs of seed path $p$ and disjoint dependent protection path $q$.

The TSH-DL algorithm first computes a free wavelength along the given seed path $p$ as the working path. In Step 2 it computes the dependent cost of the wavelength-links in the layered graph. To compute the dependent cost it assigns a cost of 0 to all the wavelength-links used by the protection paths $q_{i}$ whose corresponding working path $p_{i}$ is edge-disjoint with the seed path $p$. In Step 3, TSH-DL removes all the links on all the wavelengths along the seed path $p$. TSH-DL now computes a shortest path $q$ in this 
modified graph with the dependent cost. If such a path $q$ exists then $q$ is a disjoint protection path with dependent cost structure for the working path $p$.

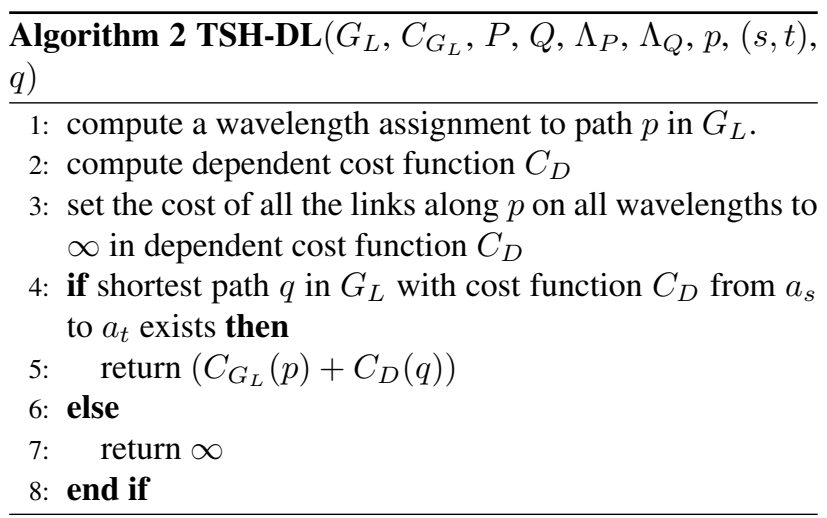

\section{EXPERIMENTAL RESULTS}

We conducted experiments on a 79-node network with 108 links and 20 wavelengths. We simulated dynamic traffic with calls arriving into the network having Poisson distribution with mean arrival rate $\lambda$. The connections have exponential holding time with mean $1 / \mu$. The load on the network is measured as $\lambda / \mu$. In a single simulation run, we simulate 100,000 calls for a specified load.

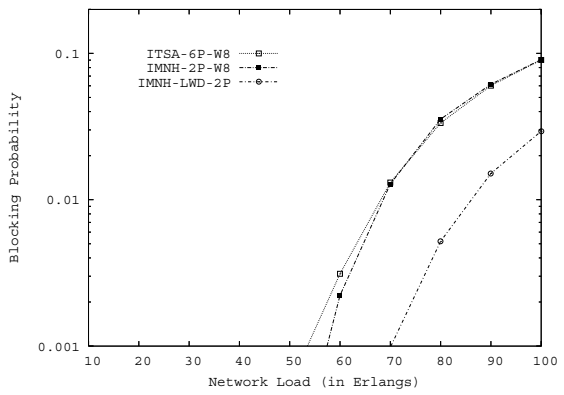

Fig. 3. Blocking Probability vs. Load (in Erlangs) of simulating 100, 000 connections on 79-node network with 20 wavelengths.

The performance of a heuristic is measured in terms of blocking probability, average working path length, average effective backup length, maximum protection capacity and maximum total capacity. However, due to space constraints we do not present the results of maximum protection capacity and total capacity. The blocking probability is the ratio of the number of blocked calls to the total number of calls simulated. The path lengths are measured in hop distance. The average working path length is the average hop length of the working paths of all the accepted connections. The effective length of a protection path of a newly arrived connection request is defined as the number of links that the protection path does not share with the protection path of any of the active connections in the network. The average effective protection path length is the average of the effective protection path lengths over all the accepted connections. The simulations are run on a lightly loaded Sun Fire V210 with 2 UltraSPARC IIIi $1 \mathrm{GHz}$ processors and 2 GB RAM.

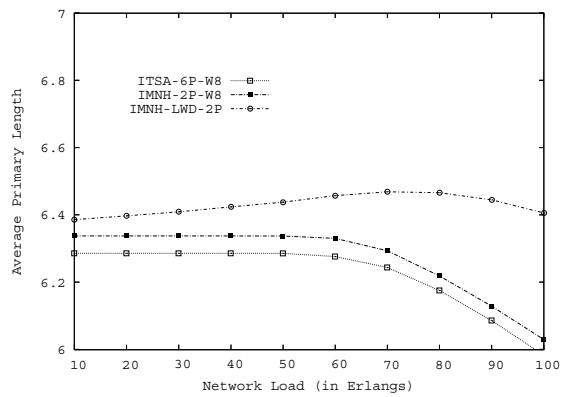

Fig. 4. The average primary length of the accepted connections during 100,000 call simulation on a 79-node network with 20 wavelengths.

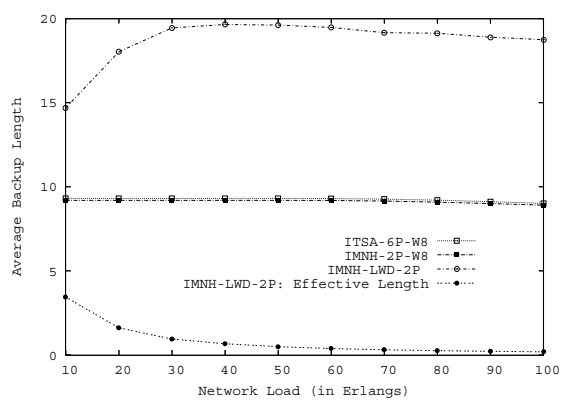

Fig. 5. The average primary length of the accepted connections during 100,000 call simulation on 79 -node network with 20 wavelengths.

We experimented with the following heuristics ITSA [4], IMNH [2], and IMNH-LWD. The Iterative Two-Step Approach (ITSA) computes disjoint working and protection paths whose sum of the asymmetrically weighted [5] costs is least. ITSA with asymmetrical weight factor of 8 and the maximum limit on the number of iterations as 6 is termed as ITSA-6P-W8. IMNH with asymmetrical weight factor of 8 and the maximum limit on the number of iterations as 2 is termed as IMNH-2P-W8. Let us term our proposed IMNHLWD heuristic with the maximum limit on the number of iterations 2 as IMNH-LWD-2P.

Figure 3 shows blocking probability with increasing network load (in Erlangs) for 100,000 call simulation using the heuristics TSH, ITSA-6P-W8, IMNH-2P-W8 and IMNH-LWD-2P. The graph clearly shows that IMNHLWD-2P outperforms all the remaining heuristics. When compared to ITSA-6P-W8, our IMNH-LWD-2P heuristic provides $67 \%$ increase in blocking performance at a network load of 100 Erlangs and an average of $74 \%$ increase in blocking performance when the network load ranges from 10 to 100 Erlangs. 
Figure 4 plots the average working path length during an entire simulation. It shows that the average working path length of IMNH-LWD-2P is longer than that of other heuristics. Figure 5 plots the average protection path length of the accepted connections among the 100, 000 connections simulated. Figure 5 also shows the effective protection path length of the accepted connections simulated using the IMNH-LWD-2P heuristic. Though the average protection path lengths for the IMNH-LWD-2P heuristic are much longer when compared to other heuristics, the average effective protection path length almost tends to 0 as network load increases. We can infer that the resources reserved for protection are efficiently utilized but at the expense of slightly longer working paths. For decreasing the average working path lengths the optimal solution can be defined as minimizing the sum of the asymmetrically weighted [5] costs of working and protection paths.

\section{CONCLUSIONS}

In this work we studied the problem of computing optimal disjoint paths in wavelength continuous networks under shared protection. We formulated the problem as computing least-cost disjoint paths with wavelength continuity constraint and dependent cost structure (LDPWCC-DCS). We introduced the concept of dependent cost of a protection path on its working path and the current status of the network. We proved that LDP-WCC-DCS problem is NP-complete. We presented an iterative heuristic (IMNH-LWD) that uses the layered graph to compute both the path and the wavelength assignment at the same time while computing the disjoint path pair. We conducted experiments on a 79-node network. When compared to ITSA, an earlier heuristic, our IMNH-LWD-2P heuristic provides $67 \%$ increase in blocking performance at a network load of 100 Erlangs and an average of $74 \%$ increase in blocking performance when the network load ranges from 10 to 100 Erlangs.

\section{REFERENCES}

[1] A. Todimala, B. Ramamurthy, N. V. Vinodchandran, "On the Complexity of Computing Least Cost Disjoint Path Pair with Dependent Cost Structure in Optical Networks," Technical Report 2005, TR05-01-03.

[2] A. Todimala, and B. Ramamurthy, "A Heuristic with Bounded Guarantee to Compute Diverse Paths under Shared Protection in WDM Mesh Networks," GLOBECOM '05, St. Louis, MO, Nov.Dec. 2005 (accepted).

[3] C. Chen, and S. Banerjee, "A New Model for Optimal Routing and Wavelength Assignment in Wavelength Division Multiplexed Optical Networks," in Proc. IEEE INFOCOM '96, 1996, vol. 1, pp. 164-171.

[4] Pin-Han Ho, "State-of-the-Art Progress in Developing Survivable Routing in Optical Metropolitan Area Networks," IEEE Communications Surveys and Tutorials.

[5] P. Laborczi, J. Tapolcai, Pin-Han Ho, T. Cinkler, A. Recski, and H. T. Mouftah, "Solving asymmetrically weighted optimal or near-optimal disjoint path pair for the survivable optical networks," in Third International Workshop On Design Of Reliable Communication Networks (DRCN '01), Oct. 2001.
[6] I. Chlamtac, A. Faragó, T. Zhang, "Lightpath (Wavelength) Routing in Large WDM Networks," IEEE Journal on Selected Areas in Communications, vol. 14, no. 5, June 1996.

[7] R. Ramamurthy, Z. Bogdanowicz, S. Samieian, D. Saha, and B. Rajagopalan, "Capacity Performance of Dynamic Provisioning in Optical Networks," Journal of Lightwave Technology, vol. 19, no. 1, pp. 40-48, Jan. 2001.

[8] G. Maier, A. Pattavina, L. Roderti, and T. Chich, "Static-Lightpath Design by Heuristic Methods in Multi-fiber WDM Networks," in Proceedings, Opticomm 2000 SPIE Conf., Oct. 2000, pp. 64-75.

[9] A. Dacomo, S. D. Patre, G. Maier, A. Pattavina, and M. Martinelli, "Design of Static Resilient WDM Mesh Networks with Multiple Heuristic Criteria," in Proc. IEEE INFOCOM '02, 2002.

[10] B. Korte, L. Lovasz, H. J. Promel, A. Schrijver, Paths, Flows, and VLSI-Layout, Algorithms and Combinatorics, Springer-Verlag, 1990.

\section{APPENDIX}

\section{A. Transformation to Layered-Graph}

The layered graph model was proposed in [3], [6] and studied in [7] for solving the routing and wavelength assignment (RWA) problem in WDM optical networks. In [8] the layered graph model was extended to multifiber layered graph for solving the RWA problem in multifiber optical networks. In [9] the multi-fiber layered graph model is used to solve routing, fiber and wavelength assignment (RFWA) problem in multi-fiber networks to optimize capacity for providing fault tolerance under both dedicated and shared protection in static environments (connection requests are known ahead of time).

Let us present the transformation of a given fiber topology graph $G=(V, E)$ to a layered graph $G_{L}=$ $\left(V_{L}, E_{L}\right), \Gamma: G \longrightarrow G_{L}$. Let $C_{G_{L}}: E_{L} \longrightarrow Z$ be the edge cost function for the layered graph $G_{L}$. The layered graph consists of two types of nodes, namely, wavelength nodes and access nodes. Formally,

$$
V_{L}=\left\{n_{i, w} \mid \forall i \in V, w \in W\right\} \cup\left\{a_{i} \mid \forall i \in V\right\}
$$

where $n_{i, j}$ 's are wavelength nodes and $a_{i}$ 's are access nodes. The edges in the layered graph are also of three types, namely, wavelength selection links, and wavelength routing links. The layered graph corresponding to wavelength continuous networks does not have wavelength conversion links. Formally the edges in layered graph corresponding to wavelength continuous networks is

$$
E_{L}=\left\{e_{i, w}\right\} \cup\left\{e_{(i, j), w}\right\}
$$

where $\left\{e_{i, w}\right\}=\left\{\left(a_{i}, n_{i, w}\right) \mid \forall i \in V, w \in W\right\}$ and $\left\{e_{(i, j), w}\right\}=\left\{\left(a_{i, w}, a_{j, w}\right) \mid \forall(i, j) \in E, w \in W\right\}$ are wavelength selection and wavelength routing links respectively. The cost of the wavelength selection links is 0 . The cost of the wavelength routing links $e_{(i, j), w}$ is the same as the cost of the link $(i, j)$ in graph $G$. The time complexity of transforming the graph $G$ to the layered graph $G_{L}$ for a wavelength continuous network is $O(n W+m W)$ where $n$ is the number of nodes and $m$ is the number of edges in the fiber topology graph $G$. 